\title{
Status of 3D Printing Technology for Metal Materials
}

\author{
Jinjian Lv, Changzhi Jia, Jianchun Yang, Yufei Zhang \\ Mechanical Engineering Colloge,Shijiazhuang 050003,China \\ 15630975586@163.com
}

\begin{abstract}
Keywords: 3D Printing,Additive Manufacturing,Metal materials,Laser
Abstract.In this paper, the basic principles of 3D printing technology was briefly introduced and several methods of 3D printing for metal materials were introduced in details, including Electron Beam Melting (EBM), Selective Laser Melting forming (SLM), Selective Laser Sintering forming (SLS), Laser Direct Melting Deposition (LDMD). Then, the application field of 3D printing for metal materials and its development were briefly introduced. At the end of this paper, combined with the status of 3D printing for metal materials, it was pointed out that $3 \mathrm{D}$ printing for metal materials should focus on printing powder, 3D printing equipment for metal materials, 3D printing parts nondestructive testing methods, 3D printing parts failure behavior and life prediction, and the establishment of non-destructive testing for 3D printing parts of the standard and the database of $3 \mathrm{D}$ printing materials comprehensive mechanical properties.
\end{abstract}

\section{Introduction}

3D printing technology is also said to Additive Manufacturing, is relative to the traditional machining and other "material manufacturing" technology that is based on the discrete / accumulation principle, through the gradual accumulation of materials to achieve manufacturing technology ${ }^{[1,2]}$. The emergence of 3D printing technology to the development of industrial products, handicrafts, model design provides the most convenient manufacturing tools, the development speed of the product several times to improve, and the more complex structure of the object, its manufacturing speed was significant. Into the 21 st century, by material manufacturing technology and its application has been rapid development. Compared with the traditional manufacturing technology, incremental material manufacturing technology has obvious technical advantages, but there are limitations ${ }^{[3]}$. 3D printing technology is a typical dual-use technology, its wide range of applications, including: aerospace, weapons and equipment, industrial design and manufacturing, mold, medical and fashion, film, architecture, creative design and many other different industries. According to the 3D printing technology used in the form of materials and forming methods, 3D printing technology can be broken down into a variety of technologies, including metal materials, 3D printing technology research is the most attention of the direction of metal materials, 3D printing technology for industrial change is epoch-making significance ${ }^{[4]}$. Restrictions on metal materials, 3D printing technology, many factors such as laser light path system, control software, mechanical precision, oxygen content control, powder preparation process. This paper mainly introduces the research and application of 3D printing technology of metal materials.

\section{The classification of 3D Printing Technology for Metal Materials}

$3 \mathrm{D}$ printing technology can be divided into three type ${ }^{[5-7]}$ : Electron Beam Melting (EBM), Selective Laser Melting (SLM), Selective Laser Sintering (SLS), Laser Direct Melting Deposition (LDMD).

\section{Electron Beam Melting}

Electron beam melting molding technology is a vacuum environment in the electron beam as a heat source to metal powder as the forming material, by constantly spreading the metal powder in the powder bed and then scan the electron beam melting, so that a small pool of mutual fusion and solidification, So continue to form a complete metal parts entity. This technique allows the formation of complex metal parts with excellent performance, but the forming dimensions are limited by the powder bed and the vacuum chamber. 


\section{Selective Laser Melting}

The principle of selective laser melting is similar to that of electromagnetism electromagnetism. It is also a kind of powder forming technology based on powder bed, but the heat source is replaced by the electron beam and the laser beam can be formed. By this technique, Performance, good surface quality of the metal parts, but the current technology cannot shape a large-size parts.

\section{Selective Laser Sintering}

The principle of selective laser sintering is similar to that of selective laser melting. It is also a kind of powder forming technology based on powder bed, but the materials used are different. The material used in the selective laser melting is a single material with small particle size ${ }^{[8]}$. However, the material used in the selective laser sintering technology is composed of a high melting point functional material and a low melting point bonding material. This technology than the selection of laser melting technology forming speed, but the roughness of forming parts, low density.

\section{Laser Direct Melting Deposition}

Metal laser melting deposition technology to laser beam as the heat source, through the automatic powder feeding device to synchronize the metal powder, precision into the laser forming surface in the formation of molten pool ${ }^{[9]}$. As the laser spot moves, the powder is continuously fed into the bath to melt and then solidify, resulting in the desired shape. This forming process can form large-size metal parts, but can not be formed very complex parts.

\section{Research Status of 3D Printing Technology for Metal Materials}

Nearly 30 years, 3D printing technology has made rapid development, the types of materials used more and more, more and more complex forming structure, parts of the increasingly high precision, so that the application of 3D printing technology continues to expand ${ }^{[10]}$. Metal material 3D printing is one of the most advanced parts of 3D printing technology. It is rapidly expanding in aerospace, medical and other fields, and has great development potential in the future.

\section{Research Status of 3D Printing Technology for Metal Materials}

AeroMet in 2002 to 2005 between the 3D printing technology through the preparation of the joints, the keel web, pylon ribs, thrust beam, wing root rings, with ribs and other aircraft parts; United States San-dia National Laboratory of the use of the technology carried out stainless steel, titanium, high temperature alloys and other metal materials, 3D printing research, and the successful realization of a satellite TC4 titanium alloy parts rough forming. The forming process takes considerably less time than conventional methods.

In the 1990s, Germany Fraunhofer Institute proposed the use of laser selective melting (SLM) method of printing metal materials, and in 2002 the success of the study. Then the company launched a number of SLM equipment, such as MCP MCPeal developed by the system, EOS developed EOSINTM series, RENISHAW AM250 system developed by the companyy ${ }^{[11]}$. In addition, a number of foreign universities and research institutes such as the German Aachen University of Technology, the University of Leeds, Liverpool University, Belgium Rubin University, Osaka University and the British Welding Institute, Germany Fraunhofer Laser Technology Research Institute, Germany, and other research institutions and Boeing, Lockheed - Martin, EADS and other large aerospace companies funding, the SLM material properties, defect control, stress control and other basic issues carried out a lot of research work 3 metal powder research status ${ }^{[12]}$.

In recent years, EBM technology in the field of aerospace development is very rapid, a number of airlines have carried out the use of EBM technology for the manufacture of complex parts of the aero engine research, which Italy AVIO company successfully using the technology to prepare the TiAl-based alloy engine blades, Has aroused widespread concern in the aviation industry.

\subsection{Research Status of 3D Printing Technology for Metal Materials of China}

The 20th century, 90 years, Northwestern Poly technical University, Beijing University of Aeronautics and Astronautics, and other universities began on the laser rapid prototyping technology research ${ }^{[13]}$. Northwestern Poly technical University has established a laser rapid prototyping system for a variety of metal materials to carry out the process of experiment, in recent years, Xigong large team using 3D printing technology to print the maximum size of $3 \mathrm{~m}$, weighing $196 \mathrm{~kg}$ aircraft titanium alloy left upper edge; In the large-scale titanium parts 3D printing carried out in-depth study in the "Eleventh Five-Year" period, the use of laser melting deposition method to prepare a large titanium bearing the main bearing structural parts; Huazhong University of Science and Technology in the laser selection of melting and laser Selection of sintered areas to carry out a lot of work on metal materials and polymer materials, 3D printing was studied and developed with independent intellectual property rights SLPM equipment HRPM series of powder melting and forming equipment ${ }^{[14]}$; Xi'an Jiaotong University in biomedical use of the 3D Printing and laser melting deposition of metal materials, and completed a 
number of cases of orthopedic 3D print personalized repair of clinical cases, prepared by laser melting of the engine blade prototype, the thinnest of up to $0.8 \mathrm{~mm}$, and has a directional crystal Organizational structure.

In addition to domestic universities, many research institutes are also carrying out 3D printing technology research. Northwest Research Institute of Nonferrous Metals in the electron beam selection process of melting technology and equipment research and development, and carried out titanium alloy, TiAl alloy electron beam melting and forming process research[14]; China Aviation Industry Corporation Beijing Institute of Aeronautical Manufacturing Engineering carried out the electronic Beam fuse deposition forming research work, and have such equipment research and development capabilities, using this method has been formed in the $2100 \mathrm{~mm} \times 450 \mathrm{~mm} \times 300 \mathrm{mmd}$ titanium bearing the main bearing structural parts ${ }^{[15]}$.

\section{Issues that need to be resolved of 3D Printing Technology for Metal Materials}

The use of laser, electron beam as the heat source of 3D printing metal components, metal powder or metal wire is set according to the path of a layer of overlay stack, the final formation of the target parts, the essence of welding ${ }^{[16]}$. Therefore, 3D printing metal parts must exist within the pores, cracks, inclusions, welding and other defects are not fusion, so metal materials, 3D printing technology is one of the important issues of defect control technology. 3D anisotropy, surface quality, dimensional accuracy and uniformity of 3D printing forming metal materials are different from those of traditional casting and forging. Therefore, the mechanical properties and dimensional accuracy of 3D printing are another research focus ${ }^{[17]}$. In addition, how to improve print efficiency and reduce the cost of mass production, is also an urgent need to solve the problem. In particular, the 3D printing technology of metal materials should improve the quality and yield of 3D printing powder, improve the capability of 3D printing equipment and develop the process monitoring system, standardize the non-destructive testing of 3D printing parts, establish the comprehensive mechanical properties of 3D printing materials Database, the study of 3D printing material failure behavior and life expectancy in several aspects to focus on.

\section{Conclusion}

3D printing is an advanced manufacturing technology, which provides a new manufacturing method for materials and structures, which is an important complement to the traditional manufacturing technology system. Especially the short process, which is suitable for the complex structure. It provides the material and structural designers with a rich imagination space, which makes the structure difficult to be realized by the traditional manufacturing technology. However, any technology has limitations, 3D printing is no exception, based on molten metal to achieve the metal structure of 3D printing, it is more suitable for plastic good metal materials, 3D printing difficult to weld metal materials, there are many difficulties, but also need Extensive research and validation. Casting, forging, welding and other metal materials manufacturing technology after hundreds of years of research, application and development, has accumulated a wealth of experience, formed a perfect standard system. 3D printing also requires a long process of accumulation and verification, only through long-term, a large number of applied research, discovery and solutions inherent in the problem, to make $3 \mathrm{D}$ printing technology to the breadth and depth of development.

\section{Acknowledgements}

This research was supported by the Science and Technology Project of Hebei Province(No.15210701D). All support is gratefully acknowledged. And I would like to express my gratitude to all those who have helped me during the writing of this thesis. I gratefully acknowledge the help of my supervisor Professor Jia Changzhi.

\section{References}

[1] Xinhong Xiong, Haiou Zhang,etal.Hybrid plasma deposition and milling for an aero engine double helix integral impeller made of super alloy [J]. Robotics and Computer Integrated Manufacturing, 2016, (26): 291-295. 
[2] Fraunhofer Institute for Production Technology IPT Controlled Metal Build Process for Generation Modification and Repair of Molds and Dies[M]. Mold Making Technology, 2006.

[3] Prof.Dr.-lng.Fritz Klocke.Rapid Prototyping and Rapid Tooling[R]. Fraunhofer Institute for Production Technology IPT, 2003.

[4] Palash Kumar.Application of rapid prototyping and rapid tooling for development of patient specific ranio facial implant: an investigative study[J]. Additive Manufacturing Technology,2008(36):510-515.

[5] Christopher B.Williams ,Joe K. Additive manufacturing of metallic cellular materials via three-dimensional printing[J]. Int J Adv Manufacturing Technology,2011(53):231-239.

[6] Weiss L E,Neplotnik G,Prinz FB,etal.Shape Deposition Manufacturing of Wearable Computers Solid Free form Fabrication Symposium[D].The University of Texas At Austin, 1996.

[7] A.G.Cooper,S.Kang,etal.Automated fabrication of complex molded parts using Mold Sha-pe Deposition Manufacturing [J]. Materials and Design,1999(20):83-89.

[8] K.P.Karunakaran,S.Suryakumar,etal.Low cost integration of additive and subtractive processes for hybrid layered manufacturing $[\mathrm{J}]$. Robotics and Computer-Integrated Manufacturing,2010,26:490-499.

[9] Sreenathbabu Akula, K.P. Karunakaran. Hybrid adaptive layer manufacturing:An Intelligent art of direct metal rapid tooling process [J]. Robotics and Computer-Integrated Manufacturing,2006(22):113-123.

[10] Jeng-Ywan Jeng,Mingching Lin. Mold fabrication and modification using hybrid processes of selective laser cladding and milling [J].Journal of Materials Processing Technology,2011(110):98-103.

[11] Wohlers Associates. Additive manufacturing and 3D printings state of the Industry [J]. Annual Worldwide Progress,2013(16):21-27.

[12] J.M.Pinilla,F.B.Prinz.Lead-time reduction through flexible routing:application to Shape D-eposition Manufacturing [J]. International Journal of Production Research,2013,13:2957-2973

[13] Prof.Dr.-lng.Fritz Klocke.Rapid Prototyping and Rapid Tooling[R]. Fraunhofer Institute for Production Technology IPT,2003.

[14] Mohammad Pervez Mughal. The effects of machining on material properties in hybrid, welding/milling based rapid prototyping [J].Computational Materials Science and Surface Engineering,2009,2(1/2):99-102.

[15] $\mathrm{Zhu} \mathrm{Hu}$, Kunwoo Lee. Determination of optimal build orientation for hybrid rapid prototyping[J].Journal of Materials Processing Technology,2012:378-383.

[16] Min Li. The Application of Rapid Prototyping Technique in Chin Augmentation[J]. Aesth Plast Surg,2010(34):172-178.

[17] Min-Saeng Kim. Direct Metal Printing of 3D Electrical Circuits Rapid Prototyping [J]. International Journal of Precision Engineering and Manufacturing,2009.10(5):147-150. 